\title{
Lifestyle-oriented non-pharmacological treatments for fibromyalgia: a clinical overview and applications with home-based technologies
}

This article was published in the following Dove Press journal:

Journal of Pain Research

24 October 2012

Number of times this article has been viewed

\section{Fred Friedberg' \\ David A Williams ${ }^{2}$ \\ William Collinge ${ }^{3}$}

'Department of Psychiatry and Behavioral Science, Stony Brook University, Stony Brook, New York ${ }^{2}$ Department of Internal Medicine, University of Michigan, Ann Arbor, Michigan, ${ }^{3}$ Collinge and Associates, Kittery, Maine, USA
Correspondence: Fred Friedberg

Putnam Hall/South Campus,

Stony Brook University, Stony Brook,

NY I I794-8790, USA

$\mathrm{Tel}+\mid$ 63| 6328252

Fax + I 63I 6323165

Email fred.friedberg@stonybrookmedicine. edu

\begin{abstract}
Fibromyalgia (FM) is a persistent and disabling widespread pain condition often accompanied by chronic fatigue, cognitive problems, sleep disturbance, depression, anxiety, and headache. To date, the most thoroughly studied non-pharmacological approaches to managing FM are those with a focus on changing patient activities and beliefs that affect the illness. These interventions are intended to facilitate enduring improvement in pain and functional status. Lifestyle-oriented treatments include patient education, aerobic or other physical exercise, and cognitive-behavioral therapy (CBT). These interventions in FM can be delivered in medical or behavioral health care settings by trained professionals, through patient-oriented treatment manuals, or via remote-access technologies. Non-pharmacological treatments, in particular exercise and CBT, have yielded effect sizes and cost-benefit ratios comparable to medications. This paper describes lifestyle-oriented non-pharmacological treatments for FM and highlights selected literature reviews of these interventions. In addition, behavioral and practical issues are addressed that may affect these non-pharmacological treatments, including patient expectations, participant burden, and treatment availability. Recommendations are made to facilitate these interventions and potentially improve outcomes. In particular, the increasing availability of convenient home-based mobile technologies to deliver these non-pharmacological treatments is described.
\end{abstract}

Keywords: cognitive-behavior therapy, exercise, education, mobile technology

\section{Introduction}

Fibromyalgia (FM) is a persistent and disabling widespread pain condition often accompanied by chronic fatigue, cognitive problems, sleep disturbance, depression, and anxiety. The functional limitations reported by patients include reduced exercise tolerance and pain exacerbations caused by the activities of daily living. ${ }^{1}$ In a population study ${ }^{2}$ of ten common chronic illness conditions, FM was the highest ranked for (1) needed health care not received in the previous year as well as (2) long-term disability, pain, and poor self-rated health. In addition, health care utilization and costs for FM have been estimated at US\$6000 per patient per year by the Centers for Disease Control and Prevention, ${ }^{3}$ and an astonishing US\$18,000+ annually in a recent administrative claims study of over 5000 FM patients. ${ }^{4}$

\section{Mechanisms}

Current evidence suggests that centrally augmented pain processing and deficiencies in descending central modulation ${ }^{5}$ play an important role in producing the debilitating pain experienced by patients with FM. Mechanisms underlying central augmentation 
may produce pain exacerbation and also negatively influence sleep, mood, memory, and level of alertness. ${ }^{5}$ This could account for the clustering of symptoms commonly found in FM. The illness is also associated with depression, anxiety, and stress. ${ }^{6,7}$ No biological diagnostic tests have been established for FM. The current clinical diagnostic criteria overlap symptomatically with many other conditions, particularly chronic fatigue syndrome and migraine headache. ${ }^{8}$

\section{Management}

Given the complex symptom presentation and multiple comorbidities associated with FM, it is unlikely that the typical patient will be successfully managed by a single practitioner. Preferably, FM is treated by a multidisciplinary team whose members are variously expert in a variety of physical, cognitive, behavioral, and educational strategies. ${ }^{9}$ Given that such multidisciplinary teams may not be available in all communities, treatment by a primary care physician in conjunction with targeted referrals to nonmedical specialists may be a more pragmatic approach.

Medically, three US Food and Drug Administrationapproved medications are available for FM. Two are classified as serotonin/norepinephrine reuptake inhibitors - duloxetine ${ }^{10}$ and milnacipran ${ }^{11}-$ and the other is an anticonvulsant - pregabalin. ${ }^{12}$ About $40 \%$ of patients experience a clinically important reduction in pain with these new drugs. ${ }^{13}$ Yet non-pharmacological treatments, in particular exercise and cognitive-behavior therapy (CBT), have yielded effect sizes and cost-benefit ratios comparable to medications. ${ }^{14,15}$

To date, the most thoroughly studied and efficacious nonpharmacological approaches to managing FM are those with a focus on changing unhelpful beliefs/attitudes and activities associated with the illness. While other non-pharmacological interventions may have benefit for specific individuals (eg, massage, nutritional approaches), these interventions lack sufficient evidence from randomized controlled trials to establish efficacy. ${ }^{16}$

Efficacious lifestyle-oriented interventions include patient education, aerobic or other physical exercise, and CBT. ${ }^{8}$ These approaches to the management of FM can be delivered in medical or behavioral health care settings by trained professionals, through patient-oriented self-management manuals, or via the Internet or mobile technologies. ${ }^{17}$ Despite their demonstrated efficacy, a number of challenges prevent the broader adoption of these interventions. These challenges include low compliance and high dropout rates for exercise, ${ }^{18}$ loss of benefit at follow-up if compliance is low, ${ }^{19}$ and, more generally, a lack of availability of these interventions to many patients. ${ }^{20}$

\section{Specific aims}

This paper is intended for the health practitioner seeking clinically useful information on evidence-based nonpharmacological treatment of the FM patient. Often practitioners are quite familiar with biomedical approaches to FM but less comfortable with the non-pharmacological options recommended in clinical guidelines (eg, the APS Guideline for the Management of Fibromyalgia ${ }^{21}$ ). This paper describes lifestyle-oriented non-pharmacological treatments for FM and highlights selected literature reviews supporting the use of these interventions. Based on a combination of the published evidence and the authors' own extensive clinical experience, practical recommendations are made for the implementation of these lifestyle interventions.

In addition, we will briefly address behavioral and practical issues that may influence the success of these nonpharmacological treatments, including patient expectations, participant burden, and treatment availability. The final section of the paper is devoted to home-based technologies that can potentially expand the availability of these behavioral management options.

\section{Expectations for pain treatments}

Perhaps an implicit assumption shared by patients and their care providers is that persistent pain can be eliminated or cured. ${ }^{22-24}$ Although permanent eradication of clinical pain may be a long-range research goal, few, if any, FM patients reach a pain-free status via therapy or other means. Even for those who do report substantial reductions or "recovery" with respect to pain symptoms, long-range maintenance of such improvement often depends on carefully managing lifestyle to avoid or reduce pain triggers such as stress and over-exertion. ${ }^{25}$

For instance, an interesting qualitative study conducted on five women who reported "recovery" from $\mathrm{FM}^{26}$ found that a particular cognitive strategy was useful in pursuing activities related to their recovery status. Specifically, these subjects used pain as a warning signal of too much stress in their lives, rather than viewing pain simply as a symptommanagement issue. This "warning signal" approach greatly enhanced their ability to alter their life goals and everyday obligations in order to maintain their improved status. Thus, "recovery" was not equivalent to eradication of symptoms but, rather, to symptom remission maintained by identifying early pain and stress triggers and then making lifestyle adjustments to prevent exacerbations. 
Prior to treatment, patients' expectations, regardless of whether they are recovery focused, should be identified so that they may be better aligned with the benefits that are and are not possible. In a recent study of patient expectations in FM and back pain, ${ }^{27} \mathrm{FM}$ patients had high standards for success ( $>50 \%$ reductions in pain and fatigue and interference). Yet, both FM and back-pain patients did not expect treatment to meet their success criteria. Assessing patients' success standards and treatment expectations encourages discussion and the development of more realistic individualized treatment goals.

Given that complete pain relief is an unlikely outcome, the use of acceptance-based approaches (ie, acceptance and commitment therapy) for pain management may be useful in conjunction with any form of intervention. A number of studies have shown that greater acceptance of chronic pain is associated with better emotional, physical, and social functioning. ${ }^{28}$

Common features of acceptance beliefs in chronic pain include an acknowledgment that a cure for pain is unlikely, a shift in focus away from pain to non-pain aspects of life, and a resistance to any suggestion that pain is a personal weakness. A small qualitative study of FM and rheumatoid arthritis patients ${ }^{29}$ found evidence for acceptance beliefs prior to a 1-week self-management program. These beliefs, expressed as personal goals for self-management, included: empowerment to assume more responsibility for their health and self-care, improving self-acceptance, and helping to retain employment for those who were working.

\section{Patient education}

Patient education programs typically involve individual and/or group sessions facilitated by health professionals familiar with FM. These programs convey information about symptoms, course of illness, comorbid conditions, potential causes of the illness, psychosocial factors that influence pain, current pharmacologic and non-pharmacologic treatments, and self-management instruction. ${ }^{30}$

Studies of educational programs ranging in duration from 1.5 days to 6 months $^{31}$ have had mixed success with some showing functional or symptomatic improvements in comparison to control conditions. ${ }^{30}$ Often educational protocols are one element of larger multimodal programs that contain active intervention components (eg, interactive self-management focusing on exercise). ${ }^{32}$ Education appears most effective when included in behaviorally activating multimodal interventions. ${ }^{25}$ For example, a controlled study $^{33}$ found that self-management-oriented education in
FM significantly increased the efficacy of group exercise interventions (progressive walking, simple strength training movements, and stretching activities).

Clauw and Crofford ${ }^{20}$ suggest that a primary care setting may be especially suited to providing education and clinical care for these patients, given the high frequency of visits required and the important influence of psychosocial issues on the FM patient. A large randomized trial of patient education conducted in primary care consisted of nine 2-hour group sessions (five sessions of education and four sessions of autogenic relaxation). ${ }^{30}$ The speakers included four general practitioners and one rheumatologist. Patients were encouraged to be active, to ask questions, and to discuss issues with the speakers or with other participants. Usual care plus psycho-education produced a greater increase in patients' functional status than usual care alone. Specific improvements were seen in physical function, days feeling well, pain, general fatigue, morning fatigue, stiffness, anxiety, and depression.

\section{Recommendation}

When the patient is receptive to learning self-management, an educational multi-professional or multimodal program is an important initial step toward engaging the patient in activities that will have a therapeutic effect on health and quality of life. If this type of program is not available, an informed physician can provide patient education over multiple visits ${ }^{33}$ and refer to specialists for more focused non-pharmacological treatments.

\section{Physical exercise}

Exercise is one of the most commonly recommended nonpharmacological strategies for the management of FM. ${ }^{34}$ In a comprehensive systematic review and meta-analysis ${ }^{35}$ of randomized controlled trials evaluating exercise in FM, aerobic and mixed exercise interventions improved physical function and depression. Another review of exercise in $\mathrm{FM}^{36}$ found substantial similarity in the effectiveness of land- or water-based physical aerobic exercise and muscle strengthening exercise in improving aerobic physical fitness and functional state.

Water-based aerobic exercise offers some advantages over similarly intense land-based aerobic exercise in reducing spontaneous pain and improving depressive symptoms, but patients typically have to travel to a special facility to undertake water-based exercise. Finally, a combination of aerobic exercise, strengthening, and flexibility has also been shown to improve psychological health status and health-related 
quality of life in patients with FM. ${ }^{37}$ As FM sufferers who consistently exercise are most likely to maintain initial benefits, ${ }^{38}$ motivating patients to continue regular exercise is a critical aspect of the intervention.

Despite the strong empirical evidence for exercise benefits in patients with FM, poor compliance and high dropout rates are evident in many exercise studies. ${ }^{1,39}$ In a systematic review and meta-analysis of randomized controlled trials of aerobic exercise in $\mathrm{FM},{ }^{18} 16$ studies reported attrition rates ranging from $27 \%$ to $90 \%$ (median $=67 \%$ ). Attrition and compliance may be more problematic with exercise protocols that carry higher levels of participant burden (eg, high-impact aerobics) as well as greater adverse effects, particularly postexercise pain. ${ }^{36}$ In addition, after the supervised phase of an exercise program, declines in exercise adherence occur, often accompanied by worsening of FM symptoms. ${ }^{40}$

\section{Recommendation}

The exercise parameters offered in this section are informed by the mentioned literature reviews with particular issues (eg, post-exertional pain) addressed by relevant clinical trials and treatment-focused clinical papers. Exercise needs to be tailored to the patient's starting fitness level and symptom severity so that she/he will not be discouraged or harmed by the activity. ${ }^{41}$ This may include strategies to integrate exercises into existing lifestyles.

\section{Higher-functioning patients}

Patients with FM who have good baseline physical function (eg, are able to work at least part-time and take care of some family responsibilities) may be able to participate in group exercise programs offered to healthy individuals. Moderateto high-intensity exercise can improve aerobic capacity and muscle tenderness in patients who can manage that level of exercise. ${ }^{41}$ Supervised group exercise may be preferable to home-based exercise, especially at initiation, as it usually provides advice on optimal exercise intensity that lessens interference from baseline pain and minimizes post-exercise pain. It also conveys the social support and encouragement to continue regular exercise. ${ }^{33}$

\section{Lower-functioning patients}

It is important to note that the ability of more severely ill patients (eg, on disability, largely homebound) to perform presumably healthy therapeutic tasks such as a 20-30-minute moderate daily walk may be quite limited due to debilitating post-exertional pain, ${ }^{42}$ long periods of inactivity, and more severe disabilities and symptoms in general. To increase the patient's tolerance for sustained physical activity, very gradual increments in walking, stretching, or other convenient low effort activity should be individualized and assigned over several weeks. Graded activity homework may begin with daily 1 - to 10 -minute walks. As activity tolerance improves, walking can be increased in one to five 5-minute increments over several weeks until daily walks reach 10-30 minutes, 5-7 days a week, by the end of treatment. ${ }^{43}$ If assigned in such small measured steps, symptom exacerbations from assigned exercise are likely to be minimal.

Relevant to this stepwise approach, a review of exercise treatment studies in $\mathrm{FM}^{44}$ concluded that the greatest effect and lowest attrition occurred in exercise programs that were of lower rather than higher intensity. In addition, Häuser et $\mathrm{al}^{18}$ reported in a meta-analysis that land- or water-based aerobic exercise of slight to moderate intensity at a frequency of two or three times per week appeared to be most effective. Positive effects were evidenced for depressed mood, healthrelated quality of life, and physical fitness, which was often maintained at follow-up (range: 3-17 months).

For some proportion of patients with FM, the word "exercise" may have negative connotations perhaps attributable to general inactivity and low fitness, ${ }^{41}$ post-exercise pain, ${ }^{36}$ or lack of desire to exercise. Yet these patients, even though quite ill, typically do at least several minutes of obligatory walking daily to perform routine tasks. ${ }^{45}$ Additional assigned minutes of voluntary walking or other comparable activity appear, by contrast, to invoke positive feelings related to stress relief and meaningful leisure activity. ${ }^{46,47} \mathrm{~A}$ focus on voluntary enjoyable activities, rather than "required" activities or vigorous exercise, may be more acceptable to many patients because the activity is framed in a more positive way, for example, "easy" walking rather than fitness-oriented walking.

\section{CBT}

The term "CBT" refers to a class of treatments grounded in a common theoretical framework. CBT combines two forms of therapy, behavioral ${ }^{48}$ and cognitive. ${ }^{49}$ Cognitive therapy examines the thinking process that influences one's perception of pain as well as one's responses to the presence of pain. Beliefs, expectations, and attributions all contribute to emotional responses that can exacerbate and perpetuate pain intensity, suffering, and diminished functioning. Behavioral therapy, based upon operant conditioning, classical conditioning, and social learning theory, helps individuals to respond more adaptively to persistent pain. CBT has also been applied to psychiatric factors that can influence chronic pain. 
For example, depression has been shown to co-occur with pain $52 \%$ of the time when assessed in pain clinics, $27 \%$ of the time when assessed in primary care clinics, and $18 \%$ of the time in population-based studies. ${ }^{50}$ In addition, anxiety disorders occur $23 \%$ of the time with pain and depression ${ }^{51}$ and personality disorders tend to occur with greater frequency in chronic pain conditions (ie, $51 \%-58 \%^{52,53}$ ) than in the general population $(15 \%) .{ }^{54}$ Thus, referral to a mental health professional may be useful to assess and treat comorbid psychiatric disorders or to help identify the role of psychological factors in chronic pain.

However, the value of CBT is not limited to treating psychiatric issues. With advances in the disciplines of health psychology and behavioral medicine, the basic tenets of CBT have been utilized to engender direct pain relief and improvements in functional status and quality of life. Cognitive- and behavioral-based approaches to pain management now have a strong evidence base that supports their efficacy for the management of chronic pain generally, and FM specifically. ${ }^{14,55-58}$

The practice of CBT for chronic pain conditions such as FM usually involves three phases. ${ }^{59}$ Phase 1 involves education, where a model for understanding how pain works and the need for patients to participate in pain management is emphasized. Phase 2 involves skills training and phase 3 applies these skills in progressively more challenging reallife situations. Homework for practicing and learning these skills is a common element of CBT, as is the use of booster sessions to help maintain the use of skills over the long-term.

\section{Phase I: education}

FM can be a confusing pain condition for which conflicting and often inaccurate information is provided by public sources, other patients, and even some clinicians. Thus, it is important that the therapist convey to the patient what is known about FM, including its possible causes and maintaining factors, and why patients need to participate in their own pain management. Good rapport with a shared understanding of the goals of CBT is also important because behavioral approaches may initially seem counterintuitive and even counterproductive until tried with success. For example, behaviors appropriate for acute pain management (eg, resting, reducing social contact) may be maladaptive if pain persists over the long-term. The education offered here in phase 1 is often similar to that already described in the "Patient education" section. However, CBT tends to go beyond simple education by offering specific skills, homework assignments, and tailored support to patients as they learn new and often challenging ways of adapting to chronic pain.

\section{Phase 2: CBT skill sets}

The CBT skills for pain management can reduce pain and improve functional status. Depending upon the specific needs of the patient, the following skill sets are often used in CBT for FM.

\section{Skills targeting pain reduction}

Teaching the relaxation response is a commonly applied form of behavioral treatment for the management of pain. ${ }^{60}$ The goal is to progressively learn what the individual needs to do to produce a profound sense of relaxation in both physically and mentally by their own direct control. A variety of methods can be used to arrive at the desired state of relaxation (eg, progressive muscle relaxation, visual imagery, hypnosis, biofeedback). Regardless of method, repeated practice is essential to successfully train the body to reduce physiological arousal and generate relaxation and quiescence. It is important to match the particular relaxation technique to the patient's preferences for ease of use and effectiveness.

\section{Skills to improve functional status}

Several studies suggest that the primary benefit of CBT for patients with FM is improvement in functional status. ${ }^{61,62}$ Behavioral skills that can be taught to increase functional status include graded-activation and pleasant activity scheduling.

Graded-activation, also known as "time-based pacing,",63 is a structured method for gradually increasing daily function. Individuals with chronic pain will often engage in greater amounts of activity than their bodies can tolerate on less symptomatic "good days." This over-activity is in part driven by a desire to get things done and to bolster a faltering self-esteem. Although personally rewarding in the short-term, such over-activity can lead to debilitating symptom flare-ups, activity crashes, lost productivity, and (paradoxically) diminished self-esteem.

Time-based pacing helps to manage this "push-crash" cycle of over-activity and collapse. By first determining how long a specific task can be done without a flare-up of pain, a "safe" time interval is established. Then the task (eg, laundry, typing, raking leaves) is done only for the duration of the safe interval followed by a rest period. The relatively brief restful pause indicates that the patient is recovering from a pre-determined safe amount of activity, rather than an unhealthy interval of symptom-producing over-activity. 
This safe pattern can be repeated many times until the task is completed. With continued practice, tolerance of physical activity will improve and the safe zone can be expanded to gradually include greater functional time. As rest periods are based upon time scheduled for an activity, rather than the occurrence symptom flare-ups, individuals actually get more done during the course of a day.

The use of time-based pacing may seem counterintuitive to patients who believe they will accomplish more by attempting to work through pain flares. Yet, time-based pacing can be more effective than working through pain flares, if the patient and therapist jointly develop a plan for steadily increasing the amount of time spent on specified targeted behaviors. Time-based pacing can be used to design exercise regimens, complete work-related activities, and re-engage in pleasant activities such as social outings and sports participation.

A complementary skill to graded-activation is pleasant activity scheduling. Individuals with pain often reduce or eliminate enjoyable activities to reserve their energies for essential tasks. A lack of enjoyable physical activities in patients with FM compared with healthy controls was found in a recent study ${ }^{45}$ on self-report and actigraph-measured activity levels over 1 week. In this study, FM patients did not significantly differ from controls in their self-reported household activities but engaged in less recreational physical activities.

While cutting back on pleasurable activities may make sense to patients given the limitations of FM, this type of activity reduction may lessen opportunities to effectively manage chronic pain. Engaging in pleasant activities is a natural way to elevate mood, ${ }^{64}$ reduce pain, ${ }^{65}$ and increase confidence in one's ability to function at a higher level. This is particularly important in FM given the low levels of positive affect found in these patients. ${ }^{66}$

The term "scheduling" is important because pleasant activities must be done regularly to generate benefits. For individuals with pain, pleasant occurrences need to be given the same priority as a meeting, a doctor's appointment, or a deadline. ${ }^{62}$ Combining graded-activation with pleasant activity scheduling helps to reinforce the use of each skill and allows patients to engage in pleasant activities for longer and at diminished risk of a flare-up. Behavioral prescriptions or recommendations for pleasant, low effort activities may have the additional advantage of replacing some of the patient's obligatory activities (eg, housework). ${ }^{46}$ With a gradual re-introduction of pleasant activities, patients develop a greater tolerance and enjoyment of activity with fewer pain flares.

\section{Skills to improve sleep}

Individuals with FM may report great difficulty falling asleep, frequent awakenings by pain or discomfort, or feeling unrefreshed when waking up in the morning. Behavioral sleep strategies, if used regularly, can lead to more restorative sleep with the additional benefits of improved mood, better pain management, less fatigue, and improved mental clarity. ${ }^{67}$ Behavioral sleep strategies may be grouped into several classes. Some focus on sleep patterns and routines (eg, having regular bed and wake times) and the use of adaptive sleep behaviors (eg, attempting to sleep only when in need of sleep). Other strategies use knowledge of sleep physiology such as taking a warm bath before bedtime and allowing one's core body temperature to drop (ie, a natural cue to fall asleep). Finally, strategies that focus on making the sleeping environment more conducive to sleep (eg, optimizing the room temperature, adjusting light levels, reducing ambient sounds, increasing the comfort of the bed, avoiding stimulating foods/drinks) can lead to more restful sleep. ${ }^{67-69}$

\section{Skills targeting thoughts about pain}

Beliefs about pain can greatly influence how well an individual manages pain. For example, strong convictions of personal helplessness, believing that only a physician can bring relief, or self-rejection because of illness limitations are learned automatic thinking patterns that can hinder productive living with chronic pain. "Cognitive restructuring"49 is a type of cognitive therapy that can be used to effectively challenge these negative automatic thoughts and train individuals how to use alternative thinking to promote greater functioning and well-being. With practice, these more adaptive thinking patterns can improve pain control and facilitate higher functioning. ${ }^{70}$

\section{Other CBT skills for pain management}

While the already mentioned approaches to pain are the most commonly used, several other skills may also be helpful. Many individuals with pain face mounting life difficulties associated with their pain (eg, how to drive with pain, legal problems, family demands). Structured approaches to problem solving help individuals divide large and complex problems into smaller, more manageable challenges. ${ }^{71}$ Individuals with pain also tend to report difficulties dealing with other people. For example, spouses may become frustrated with the pain and the limited functionality of the patient, employers may become less sympathetic over time, and busy physicians may not have sufficient time to hear the many important details that a patient wishes to communicate. Thus, training 
in communication skills is often used to help individuals more effectively garner the support of others as a means of better pain management. ${ }^{72-75}$

\section{Phase 3: applying skills in real-life settings}

In educational settings, information about FM and illness self-management is usually communicated in a lecture format to patients who may or may not act on that information. In contrast, CBT involves skills-focused homework assignments that are reviewed and tailored to the life challenges facing each patient. Thus, education about the illness is more effective when applied to challenging real-life situations. In CBT, rehearsal, repetition, and refinement of skills are key to developing behavioral changes that are maintained over time. The therapist can help the patient apply each skill in progressively more challenging real-life situations. The use of booster sessions can also help to sustain improvements over the long-term.

\section{Recommendations for CBT service delivery}

CBT can be delivered in either a face-to-face format between a trained therapist and a single patient or by a therapist in a group setting. Most interventions can be completed in 6-12 sessions with additional booster sessions to reinforce long-range changes. Each session typically lasts 50 minutes for an individual session and between 1 and 1.5 hours for group sessions. As many communities do not have therapists trained to do behavioral pain intervention, obtaining such care poses a significant, perhaps prohibitive, travel problem for patients. In addition, current reimbursement structures often limit access to CBT for pain unless a coexisting mental health problem is diagnosed.

However, more convenient alternatives to access CBT are becoming available. For example, many of the skills programs mentioned can be made available by telephone contact, ${ }^{76}$ lay coaches, ${ }^{77}$ or websites to deliver standardized evidence-based content. ${ }^{15}$ As more accessible alternatives to traditional CBT delivery become widely available (see the next section), more individuals will have the opportunity to use these approaches for pain management.

\section{Treatment availability and the use of technology}

The impact of FM places demands on patients to adopt behavioral change strategies in their daily lives to help reduce or better manage symptoms. Modern information technology (IT) makes it possible to deliver effective lifestyle-oriented interventions directly to the patient with an ease of access far greater than that of just a few years ago. Not only can IT interventions provide basic information and guidelines, but they can now also offer interactive and adaptive responses for individual patients, even on a moment-to-moment basis. ${ }^{17}$ In addition, online supportive interaction with other patients is an important feature of IT that can help address the loneliness, invalidation, and lack of social support that contribute to distress in people with $\mathrm{FM}^{78}$ These developments enable patients to receive psycho-educational input and advice that is far less costly in comparison to face-to-face interactions with specialist clinicians who are much less available.

The landscape of IT devices is changing rapidly. Apart from home computers, handheld devices in the form of tablet computers are becoming more and more common. At the time of writing, $29 \%$ of US adults own a tablet or e-reader. ${ }^{79}$ More than $90 \%$ of owners use their tablets at home in the evenings and $80 \%$ use them in bed. ${ }^{80}$ Smartphones are also becoming ubiquitous and are capable of delivering the same interactions as tablets, limited only by the screen-size preference of the user.

\section{Research on IT interventions}

In a recent review of mobile-device IT in health behavior, Heron and Smyth ${ }^{17}$ described "ecological momentary interventions" (EMIs) as treatments that are provided to people during their everyday lives (ie, in real time) and in natural settings (ie, in the real world). They reviewed 27 mobile technology-based EMIs using handheld computers or mobile phones to deliver ambulatory treatment for smoking cessation, weight loss, anxiety, diabetes management, eating disorders, alcohol use, and healthy eating and physical activity. The authors concluded that EMIs can be successfully delivered, are accepted by patients, and may be efficacious for treating a variety of physical and psychological symptoms. Despite the wide variations in study design quality and outcomes reported, EMIs are nevertheless regarded as important potential contributors to longitudinal technology-enhanced assessment and treatment. ${ }^{81}$

\section{Evidence for online interventions in FM}

A systematic analysis of the quality of existing FM information websites concluded that online FM resources do not provide comprehensive and accurate information, have low quality and poor readability, and may not be usable for most people. ${ }^{82}$ Yet, some relatively recent projects have developed evidence-based IT interventions intended to serve people with FM. The earliest of these was reported 
by Lorig et al in 2008, ${ }^{83}$ who studied the efficacy of an internet-based adaptation of the Arthritis Self-Management Program (ASMP) as a resource for patients unable or unwilling to attend small-group ASMPs. The ASMP is a widely used 6-week (6-session) in-person group program geared to a primarily arthritis-focused population but is also used by patients with FM. The intervention has proven effective in changing health-related behaviors and improving health status measures. $^{84}$

The ASMP online program is administered as a workshop guided by trained leaders who interact remotely with participants, either through email or messaging on the site. Content includes education on symptom management, physician-patient communication, diet and exercise, fatigue management, and problem solving. Participants $\log$ in a minimum of three times for a total of 1-2 hours and remotely participate in all weekly activities. The activities include reading psycho-educational content, posting action plans on the bulletin boards, online discussions with other participants, and email reminders to encourage participation.

In a study of the online program, 855 patients with rheumatoid arthritis, osteoarthritis, or FM (86 with FM alone) were randomized to either an intervention or usual care (control) group. At 1-year follow-up, the intervention group had significantly improved in four of six health status measures (health distress, activity limitation, self-reported global health, and pain) as well as self-efficacy. However, patients with FM alone appeared to gain fewer benefits from the program. Further, the authors noted that the observed changes, while statistically significant, were modest.

Another approach was developed by Williams et al, ${ }^{15}$ who conducted a randomized controlled trial of an Internetbased exercise and behavioral self-management program for 118 FM patients. Experimental subjects received routine care plus access to a Web-Enhanced Behavioral Self-Management (WEB-SM) program that taught principles of cognitive and behavioral pain management. The WEB-SM program was contained on the website entitled "Living Well with Fibromyalgia," which contained 13 modules segregated into three broad segments: (1) educational lectures providing background knowledge about FM as a disease state; (2) education, behavioral, and cognitive skills designed to help with symptom management; and (3) behavioral and cognitive skills designed to facilitate adaptive lifestyle changes for managing FM.

Each module featured a video lecture by a clinician experienced in applying a selected topic with respect to FM. This talk was accompanied by written summaries of the lecture for reading or downloading, homework and self-monitoring forms for applying the behavioral strategies described in the lecture, and supplemental educational materials unique to each topic (eg, audio relaxation exercises and readings). The investigators found significantly greater improvement in those in the active WEB-SM program compared with controls in terms of pain and physical functioning, and overall global improvement at 6-month follow-up. Exercise and relaxation techniques were the most commonly used skills. The fact that these interventions were delivered without clinician contact confirms that online intervention may be efficacious for at least some proportion of the FM population.

A later online CBT study in patients with $\mathrm{FM}^{85}$ compared CBT with usual care in a 6-week intervention with a 6-week follow-up in a randomized trial. Patients in the Internet-based CBT program had lower fibromyalgia impact and tender point scores at 6- and 12-week follow-ups in comparison to those in usual care.

Finally, Collinge et a ${ }^{86}$ developed an Internet-based intervention called "SMARTLog" (SMART, Self-Monitoring and Review Tool) for people with FM. The SMARTLog is an online instrument in which subjects record daily data on their activities, health-related behaviors, stressors, and symptom levels. With repeated use (about five minutes per day, usually for at least 20 days) users built their own personal database over time. The cumulative data were analyzed using a proprietary statistical analysis program to identify personal behavioral predictors of statistically significant changes in patients' symptom levels (eg, "When you eat dinner before $7.13 \mathrm{pm}$ your pain levels are lower the next day"). When such statistical associations were found, these statements were delivered to the user in the form of a SMART Profile. A feasibility pilot study ${ }^{86}$ of the SMARTLog in $40 \mathrm{FM}$ patients found that utilization, satisfaction, and compliance were high across the test period, and higher utilization was predictive of lower anxiety and improved physical functioning and self-efficacy. ${ }^{86}$

Collinge et al (2011) followed the above study with a Phase II trial with 497 users and found that likelihood of benefit was a function of frequency of use of the program - i.e., frequency of accessing the SMARTLog and uploading daily behavior and symptom data. Specifically, moderate use (completing the SMARTLog at least three times per week for 3 months) led to clinically significant improvements in pain, memory, gastrointestinal problems, depression, fatigue, and concentration. Heavy use (completing the SMARTLog an average of 4.5 times weekly over a 5-month period) led to clinically significant improvements in all of the just-mentioned symptoms 
as well as reduced stiffness and fewer sleep difficulties. These outcomes were based on simple utilization of the SMARTLog system, irrespective of SMART Profile statements targeting specific behavior-symptom relationships. When the user's data produced specific SMART Profile "target" statements, the impact of intervention on specific symptoms was greater. The SMARTLog program will be available in the near future at www.collinge.org.

In summary, IT offers abundant opportunities for developing effective remote interventions for FM patients. Such approaches are capable of delivering substantive information and guidance, personalized feedback systems, and interactive opportunities that address the illness difficulties of these patients, who are often isolated. While only limited initial evidence is currently available on specific IT applications for FM, it appears that patients who use these well-planned interventions derive notable benefits that range from modest to moderate.

\section{Conclusion}

Currently, biomedical approaches to FM management provide modest benefits and fall short of patients' desires for relief. Given the complex symptom presentation in FM, it is likely that optimal management of this condition will involve multiple modalities. Pharmacological combined with nonpharmacological approaches, such as education, exercise, and CBT, possess the best evidence for efficacy. Delivery of nonpharmacological approaches may be difficult due to the lack of providers, poor reimbursement, and travel burden on patients. Internet-based delivery of non-pharmacological treatments may be one potential solution to making these interventions available to more individuals with FM. In addition, Internetbased delivery appears to retain the effectiveness of traditional face-to-face delivery and retains high patient satisfaction.

\section{Disclosure}

The authors report no conflicts of interest in this work.

\section{References}

1. Mease P, Arnold LM, Choy EH, et al; OMERACT Fibromyalgia Working Group. Fibromyalgia syndrome module at OMERACT 9: domain construct. J Rheumatol. 2009;36(10):2318-2329.

2. Kasman NM, Badley EM. Beyond access: who reports that health care is not being received when needed in a publicly-funded health care system? Can J Public Health. 2004;95(4):304-308.

3. Wolfe F, Anderson J, Harkness D, et al. A prospective, longitudinal, multicenter study of service utilization and costs in fibromyalgia. Arthritis Rheum. 1997;40(9):1560-1570.

4. Zhao Y, Sun P, Watson P, Mitchell B, Swindle R. Comparison of medication adherence and healthcare costs between duloxetine and pregabalin initiators among patients with fibromyalgia. Pain Pract. 2011;11(3): 204-216.
5. Phillips K, Clauw DJ. Central pain mechanisms in chronic pain states maybe it is all in their head. Best Pract Res Clin Rheumatol. 2011;25(2): $141-154$.

6. Alok RD, Das SK, Agarwal SK, Salwahan L, Srivastava R. Relationship of severity of depression, anxiety and stress with severity of fibromyalgia. Clin Exp Rheumatol. 2011;29(6 Suppl 69):S70-S72.

7. González-Ramírez MT, García-Campayo J, Landero-Hernández R. The role of stress transactional theory on the development of fibromyalgia: a structural equation model. Actas Esp Psiquiatr. 2011;39(2):81-87. English, Spanish.

8. Sarzi-Puttini P, Atzeni F, Salaffi F, Cazzola M, Benucci M, Mease PJ. Multidisciplinary approach to fibromyalgia: what is the teaching? Best Pract Res Clin Rheumatol. 2011;25(2):311-319.

9. Häuser W, Bernardy K, Arnold B, Offenbächer M, Schiltenwolf M. Efficacy of multicomponent treatment in fibromyalgia syndrome: a meta-analysis of randomized controlled clinical trials. Arthritis Rheum. 2009;61(2):216-224.

10. Cymbalta ${ }^{\circledR}$ (duloxetine) [package insert]. Indianapolis, IN: Lilly; 2010.

11. Savella (milnacipran) [package insert]. St Louis, MO: Forest Pharmaceuticals; 2010.

12. Lyrica $^{\circledR}$ (pregabalin) [package insert]. New York, NY: Pfizer; 2010.

13. Arnold, LM. Biology and therapy of fibromyalgia. New therapies in fibromyalgia. Arthritis Res Ther. 2006;8(4):212.

14. Glombiewski JA, Sawyer AT, Gutermann J, Koenig K, Rief W, Hofmann SG. Psychological treatments for fibromyalgia: a meta-analysis. Pain. 2010;151(2):280-295.

15. Williams DA, Kuper D, Segar M, Mohan N, Sheth M, Clauw DJ. Internet-enhanced management of fibromyalgia: a randomized controlled trial. Pain. 2010;151(3):694-702.

16. De Silva V, El-Metwally A, Ernst E, Lewith G, Macfarlane GJ; Arthritis Research Campaign working group on complementary and alternative medicines. Evidence for the efficacy of complementary and alternative medicines in the management of fibromyalgia: a systematic review. Rheumatology (Oxford). 2010;49(6):1063-1068.

17. Heron KE, Smyth JM. Ecological momentary interventions: incorporating mobile technology into psychosocial and health behaviour treatments. Br J Health Psychol. 2010;15(Pt 1):1-39.

18. Häuser W, Klose P, Langhorst J, et al. Efficacy of different types of aerobic exercise in fibromyalgia syndrome: a systematic review and meta-analysis of randomised controlled trials. Arthritis Research and Therapy. 2010;12(3):R79.

19. Bernardy K, Füber N, Köllner V, Häuser W. Efficacy of cognitivebehavioral therapies in fibromyalgia syndrome - a systematic review and metaanalysis of randomized controlled trials. $J$ Rheumatol. 2010;37(10):1991-2005.

20. Clauw DJ, Crofford LJ. Chronic widespread pain and fibromyalgia: what we know, and what we need to know. Best Pract Res Clin Rheumatol. 2003;17(4):685-701.

21. Buckhardt CS, Goldenberg D, Crofford L, et al. Guideline for the Management of Fibromyalgia Syndrome Pain in Adults and Children. Glenview, IL: American Pain Society (APS); 2005.

22. Taylor ML. The impact of the "business" of pain medicine on patient care. Pain Med. 2011;12(5):763-772.

23. Geisser ME, Roth RS, Williams DA. The allure of a cure. J Pain. 2006;7(11):797-799; discussion 804-806.

24. Roth RS, Geisser ME, Williams DA. Interventional pain medicine: retreat from the biopsychosocial model of pain. Trans Behav Med. 2012;2(1):106-116.

25. Henriksson CM. Living with continuous muscular pain - patient perspectives. Part II: Strategies for daily life. Scand J Caring Sci. 1995;9(2):77-86.

26. Mengshoel AM, Heggen K. Recovery from fibromyalgia - previous patients' own experiences. Dis Rehabil. 2004;26(1):46-53.

27. O'Brien EM, Staud RM, Hassinger AD, et al. Patient-centered perspective on treatment outcomes in chronic pain. Pain Med. 2010;11(1): $6-15$. 
28. McCracken LM, Vowles KE, Eccleston C. Acceptance-based treatment for persons with complex, long standing chronic pain: a preliminary analysis of treatment outcome in comparison to a waiting phase. Behav Res Ther. 2005;43(10):1335-1346.

29. Hamnes B, Hauge MI, Kjeken I, Hagen KB. 'I have come here to learn how to cope with my illness, not to be cured': a qualitative study of patient expectations prior to a one-week self-management programme. Musculoskeletal Care. 2011;9(4):200-210.

30. Luciano JV, Martínez N, Peñarrubia-María MT, et al; FibroQol Study Group. Effectiveness of a psychoeducational treatment program implemented in general practice for fibromyalgia patients: a randomized controlled trial. Clin J Pain. 2011;27(5):383-391.

31. Oh TH, Stueve MH, Hoskin TL, et al. Brief interdisciplinary treatment program for fibromyalgia: six to twelve months outcome. Am J Phys Med Rehabil. 2010;89(2):115-124.

32. Hassett AL, Gevirtz RN. Nonpharmacologic treatment for fibromyalgia: patient education, cognitive-behavioral therapy, relaxation techniques, and complementary and alternative medicine. Rheum Dis Clin North Am. 2009;35(2):393-407.

33. Rooks DS, Gautam S, Romeling M, et al. Group exercise, education, and combination self-management in women with fibromyalgia: a randomized trial. Arch Intern Med. 2007;167(20):2192-2200.

34. Robinson R, Mease P, Chen Y, et al. Treatment patterns for patients with fibromyalgia: Baseline findings from the REFLECTIONS study. J Pain. 2011;12(Suppl 4):49.

35. Häuser W, Thieme K, Turk DC. Guidelines on the management of fibromyalgia syndrome - a systematic review. Eur J Pain. 2010; 14(1):5-10.

36. Cazzola M, Atzeni F, Salaffi F, Stisi S, Cassisi G, Sarzi-Puttini P. Which kind of exercise is best in fibromyalgia therapeutic programmes? A practical review. Clin Exp Rheumatol. 2010;28(6 Suppl 63):S117-S124.

37. Sanudo B, Galiano D, Carrasco L, de Hoyo M, McVeigh JG. Effects of a prolonged exercise program on key health outcomes in women with fibromyalgia: a randomized controlled trial. J Rehabil Med. 2011;43(6):521-526.

38. Vlaeyen JW, Morley S. Active despite pain: the putative role of stoprules and current mood. Pain. 2004;110(3):512-516.

39. Kurtais Y, Kutlay S, Ergin S. Exercise and cognitive-behavioural treatment in fibromyalgia syndrome. Curr Pharm Des. 2006;12(1):37-45.

40. Bircan C, Karasel SA, Akgün B, El O, Alper S. Effects of muscle strengthening versus aerobic exercise program in fibromyalgia. Rheumatol Int. 2008;28(6):527-532.

41. Jones KD, Clark SR, Bennett RM. Prescribing exercise for people with fibromyalgia. AACN Clinical Issues. 2002;13(2):277-293.

42. Nijs J, Almond F, De Becker P, Truijen S, Paul L. Can exercise limits prevent post-exertional malaise in chronic fatigue syndrome? An uncontrolled clinical trial. Clin Rehabil. 2008;22(5):426-435.

43. Wallman KE, Morton AR, Goodman C, Grove R, Guilfoyle AM. Randomised controlled trial of graded exercise in chronic fatigue syndrome. Med J Aust. 2004;180(9):444-448.

44. Jones KD, Adams D, Winters-Stone K, Burckhardt CS. A comprehensive review of 46 exercise treatment studies in fibromyalgia (1988-2005). Health Qual Life Outcomes. 2006;4:67.

45. McLoughlin MJ, Colbert LH, Stegner AJ, Cook DB. Are women with fibromyalgia less physically active than healthy women? Med Sci Sports Exerc. 2011;43(5):905-912.

46. Friedberg F. Chronic fatigue syndrome, fibromyalgia, and related illnesses: a clinical model of assessment and intervention. $J$ Clin Psychol. 2010;66(6):641-665.

47. Jason L, Torres-Harding S, Friedberg F, et al. Non-pharmacologic interventions for CFS: a randomized trial. J Clin Psychol Med Settings. 2007;14(4):275-296.

48. Fordyce WE. Behavioral Methods for Chronic Pain and Illness. St Louis, MO: Mosby; 1976.

49. Beck AT, Rush AJ, Shaw BF, Emery G. Cognitive Therapy for Depression. New York, NY: Guilford Press; 1979.
50. Bair MJ, Robinson RL, Katon W, Kroenke K. Depression and pain comorbidity: a literature review. Arch Intern Med. 2003;163(20): 2433-2445.

51. Bair MJ, Wu J, Damush TM, Sutherland JM, Kroenke K. Association of depression and anxiety alone and in combination with chronic musculoskeletal pain in primary care patients. Psychosom Med. 2008; 70(8):890-897.

52. Fishbain DA, Goldberg M, Meagher BR, Steele R, Rosomoff H. Male and female chronic pain patients categorized by DSM-III psychiatric diagnostic criteria. Pain. 1986;26(2):181-197.

53. Polatin PB, Kinney RK, Gatchel RJ, Lillo E, Mayer T. Psychiatric illness and chronic low-back pain. The mind and the spine - which goes first? Spine (Phila Pa 1976). 1993;18(1):66-71.

54. Grant BF, Hasin DS, Stinson FS, et al. Prevalence, correlates, and disability of personality disorders in the United States: results from the national epidemiologic survey on alcohol and related conditions. J Clin Psychiatry. 2004;65(7):948-958.

55. Dixon KE, Keefe FJ, Scipio CD, Perri LM, Abernethy AP. Psychological interventions for arthritis pain management in adults: a meta-analysis. Health Psychol. 2007;26(3):241-250.

56. Hoffman BM, Papas RK, Chatkoff DK, Kerns RD. Meta-analysis of psychological interventions for chronic low back pain. Health Psychol. 2007;26(1):1-9.

57. Morley S, Eccleston C, Williams A. Systematic review and metaanalysis of randomized controlled trials of cognitive behaviour therapy and behaviour therapy for chronic pain in adults, excluding headache. Pain. 1999;80(1-2):1-13.

58. Sheinfeld Gorin S, Krebs P, Badr H, et al. Meta-analysis of psychosocial interventions to reduce pain in patients with cancer. J Clin Oncol. 2012;30(5):539-547.

59. Keefe FJ. Cognitive behavioral therapy for managing pain. Clin Psychol. 1996;49(3):4-5.

60. Integration of behavioral and relaxation approaches into the treatment of chronic pain and insomnia. NIH Technology Assessment Panel on Integration of Behavioral and Relaxation Approaches into the Treatment of Chronic Pain and Insomnia. JAMA. 1996;276(4):313-318.

61. Rossy LA, Buckelew SP, Dorr N, et al. A meta-analysis of fibromyalgia treatment interventions. Ann Behav Med. 1999;21(2):180-191.

62. Williams DA, Cary MA, Groner KH, et al. Improving physical functional status in patients with fibromyalgia: a brief cognitive behavioral intervention. J Rheumatol. 2002;29(6):1280-1286.

63. Gil KM, Ross SL, Keefe FJ. Behavioral treatment of chronic pain: four pain management protocols. In: France RD, Krishnan KR, editors. Chronic Pain. New York, NY: American Psychiatric Press; 1988: 376-413.

64. Lewinsohn PM. The behavioral study and treatment of depression. In: Hersen M, Eisler RM, Miller PM, editors. Progress in Behavior Modification. Vol 1. New York, NY: Academic Press; 1975: 19-64.

65. Dailey PA, Bishop GD, Russell IJ, Fletcher E. Psychological stress and the fibrositis/fibromyalgia syndrome. J Rheumatol. 1990; 17(10):1380-1385.

66. Zautra AJ, Fasman R, Reich JW, et al. Fibromyalgia: evidence for deficits in positive affect regulation. Psychosom Med. 2005;67(1):147-155.

67. Morin CM, Culbert JP, Schwartz SM. Nonpharmacological interventions for insomnia: a meta-analysis of treatment efficacy. $\mathrm{Am} \mathrm{J}$ Psychiatry. 1994;151(8):1172-1180.

68. Edinger JD, Wohlgemuth WK, Krystal AD, Rice JR. Behavioral insomnia therapy for fibromyalgia patients: a randomized clinical trial. Arch Intern Med. 2005;165(21):2527-2535.

69. Williams DA. Utility of cognitive behavioral therapy as a treatment for insomnia in patients with fibromyalgia. Nat Clin Pract Rheumatol. 2006;2(4):190-191.

70. Jensen MP, Turner JA, Romano JM. Changes in beliefs, catastrophizing, and coping are associated with improvement in multidisciplinary pain treatment. J Consult Clin Psychol. 2001;69(4):655-662. 
71. Nezu AM, Nezu CM, Perri MG. Problem-solving Therapy for Depression: Theory, Research and Clinical Guidelines. New York, NY: Wiley; 1989.

72. Burckhardt CS, Mannerkorpi K, Hedenberg L, Bjelle A. A randomized, controlled clinical trial of education and physical training for women with fibromyalgia. J Rheumatol. 1994;21(4):714-720.

73. Nicassio PM, Radojevic V, Weisman MH, et al. A comparison of behavioral and educational interventions for fibromyalgia. J Rheumatol. 1997;24(10):2000-2007.

74. Nielson WR, Walker C, McCain GA. Cognitive behavioral treatment of fibromyalgia syndrome: preliminary findings. J Rheumatol. 1992;19(1):98-103.

75. Redondo JR, Justo CM, Moraleda FV, et al. Long-term efficacy of therapy in patients with fibromyalgia: a physical exercise-based program and a cognitive-behavioral approach. Arthritis Rheum. 2004;51(2): 184-192.

76. Naylor MR, Keefe FJ, Brigidi B, Naud S, Helzer JE. Therapeutic Interactive Voice Response for chronic pain reduction and relapse prevention. Pain. 2008;134(3):335-345.

77. Lorig K, Feigenbaum P, Regan C, Ung E, Chastain RL, Holman HR A comparison of lay-taught and professional-taught arthritis selfmanagement courses. J Rheumatol. 1986;13(4):763-767.

78. Kool MB, Greenen R. Loneliness in patients with rheumatic diseases: the significance of invalidation and lack of social support. J Psychol. 2012;146(1-2):229-241.

79. Rainie L. Tablet and E-book Reader Ownership Nearly Double Over the Holiday Gift-giving Period. Washington DC: Pew Internet and American Life Project; January 23, 2012. Available from: http://www. pewinternet.org/ /media//Files/Reports/2012/Pew_Tablets\%20and\%20 e-readers\%20double\%201.23.2012.pdf. Accessed June 14, 2012.
80. ComputerWeekly.com Research Library. The tablet market: consumer trends and market segmentation [web page on the Internet]. London: ComputerWeekly.com; February 9, 2012. Available from: http://bitpipe. computerweekly.com/detail/RES/1328810937_667.html. Accessed June 14, 2012.

81. Bauer S, Moessner M. Technology-enhanced monitoring in psychotherapy and e-mental health. J Ment Health. 2012;21(4):355-363.

82. Daraz L, Macdermid JC, Wilkins S, Gibson J, Shaw L. The quality of websites addressing fibromyalgia: an assessment of quality and readability using standardised tools. BMJ Open. 2011;1(1):e000152. Epub July 31, 2011.

83. Lorig KR, Ritter PL, Laurent DD, Plant K. The internet-based arthritis self-management program: a one-year randomized trial for patients with arthritis or fibromyalgia. Arthritis Rheum. 2008;59(7):1009-1017.

84. Lorig KR, Mazonson PD, Holman HR. Evidence suggesting that health education for self-management in patients with chronic arthritis has sustained health benefits while reducing health care costs. Arthritis Rheum. 1993;36(4):439-446.

85. Menga G, Dupre BJ, Ganthier C et al. Fibromyalgia: Can online cognitive behavioral therapy help? [Abstract]. Arthritis Rheum. 2011;63 supp 10:937.

86. Collinge W, Soltysik RC, Yarnold PR. An internet-based intervention for fibromyalgia self-management: initial design and alpha test. Optimal Data Analysis. 2010;1(1):163-175. Available online at: http://optimaldataanalysis.com/journal.htm.
Journal of Pain Research

\section{Publish your work in this journal}

The Journal of Pain Research is an international, peer-reviewed, open access, online journal that welcomes laboratory and clinical findings in the fields of pain research and the prevention and management of pain. Original research, reviews, symposium reports, hypothesis formation and commentaries are all considered for publication.

\section{Dovepress}

The manuscript management system is completely online and includes a very quick and fair peer-review system, which is all easy to use. Visit http://www.dovepress.com/testimonials.php to read real quotes from published authors. 